\title{
Depairing Critical Current Density of a Mesoscopic Square Superconductor
}

\author{
Edson Sardella and T. M. Oliveira \\ Departamento de Física, Faculdade de Ciências \\ Universidade Estadual Paulista, Caixa Postal 473, 17033-360, Bauru-SP, Brazil
}

Received on 14 April, 2004

\begin{abstract}
In the present paper we consider the vortex lattice properties of a square superconductor such as the vortex patterns, the Gibbs free energy, the magnetization, and the depairing critical current density. We show that this last quantity shows a matching effect, that is, it shows a discontinuous behavior as a function of the applied magnetic field.
\end{abstract}

\section{Introduction}

Recent progresses in nanotechnology have made possible the fabrication of mesoscopic superconducting materials with the size comparable to the coherence length $\xi$ and the London penetration depth $\lambda$. This has motivated both experimental and theoretical physicists to investigated the magnetic properties of these small superconductors. The properties of the vortex lattice in superconductors of confined geometries change radically with respect to their features in the bulk.

The critical current density of a film of finite thickness has been investigated by Mawatari and Yamafuji [1]. They have shown that the critical current density (associated with an applied transport current), exhibits a matching effect, that is, this quantity decreases discontinuously in small steps as the magnetic field, uniformly applied to the film, increases.

More recently, one of the present authors [2] has considered a more general scenario of a film of finite cross section, namely, with finite width $a$ and also with finite thickness $b$. However, only the shape of the vortex lattice and magnetization were studied; in addition, new expressions for the free energy and the local magnetic field have been found. In the present work, we will go a little further by considering the depairing critical current density. ${ }^{1}$ In our previous work, the field distribution and energy were determined for any finite values of $a$ and $b$. So we can use those results for the case of $(a, b)$ comparable to the fundamental lengths $(\xi, \lambda)$. However, our approach has a limitation concerning the number of vortices allowed inside the sample. Because we use
London theory which supposes that the nearest neighbor distances between the vortices is much larger than the coherence length $\xi$, we are restricted only to a sufficiently small number of vortices.

Very recently, Mel'nikov et al. [3] have analyzed the vortex lattice of a square mesoscopic superconductor of very small size, $8 \xi \times 8 \xi$, in the context of Ginzburg-Landau theory. They have found some exotic configurations like vortex molecules and multiquanta vortices. Similar results have been independently reported by Doria and Zebende [4]. In our work we find only singly quantized vortex configurations because of the limitations of London theory previously mentioned.

In what follows we will first outline the theoretical model in which we will be based on. Secondly, we will discuss the numerical method we use to find the vortex configurations which minimize the free energy. Thirdly, and last, we will discuss the results for the critical fields, the vortex profiles, the magnetization, and the depairing critical current density of a square superconductor.

\section{The Theoretical Model}

Let us consider a very long cylinder of rectangular cross section of sides $a$ and $b$. The applied field is parallel to the cylinder axis. Using appropriate boundary conditions and London theory, it was found in Reference [2] that the local magnetic field is given by

$$
\begin{aligned}
h(x, y)= & \Phi_{0} \sum_{i=1}^{N} G\left(x^{\prime}, y^{\prime}, x_{i}, y_{i}\right)+H\left\{\frac{\cosh [(y-b / 2) / \lambda]}{\cosh (b / 2 \lambda)}\right. \\
& \left.+\frac{4}{b} \sum_{m=0}^{\infty} \frac{1}{\alpha_{2 m+1}^{2}} \frac{b}{(2 m+1) \pi} \sin \left[\frac{(2 m+1) \pi y}{b}\right] \frac{\cosh \left(\alpha_{2 m+1} x / \lambda\right)}{\cosh \left(\alpha_{2 m+1} a / 2 \lambda\right)}\right\},
\end{aligned}
$$

\footnotetext{
${ }^{1}$ Notice that this a more fundamental quantity than that considered in Reference [1]. It is the current density near the vortex core where it achieves its highest value. Currents above this value will break apart the superconducting Cooper pairs.
} 
where $\left(x_{i}, y_{i}\right)$ is the position of the $i$-th vortex, $\alpha_{m}^{2}=\left[1+(m \pi \lambda / b)^{2}\right], H$ is the applied field, and $G\left(x, y, x^{\prime}, y^{\prime}\right)$ is the Green's function which is given by

$$
G\left(x, y, x^{\prime}, y^{\prime}\right)=\frac{1}{b} \sum_{m=1}^{\infty} g_{m}\left(x, x^{\prime}\right) \sin \left(\frac{m \pi y}{b}\right) \sin \left(\frac{m \pi y^{\prime}}{b}\right)
$$

where

$$
g_{m}\left(x, x^{\prime}\right)=\frac{1}{2 \lambda \alpha_{m} \sin \left(\alpha_{m} a / \lambda\right)}\left\{\cosh \left[\alpha_{m}\left(\left|x-x^{\prime}\right|-a\right) \lambda\right]-\cosh \left[\alpha_{m}\left(x-x^{\prime}\right) / \lambda\right]\right\}
$$

In what follows we will work in the limit $(m \pi \lambda / b)^{2} \gg 1$. Within this limit, the summation in $m$ can be evaluated exactly. Proceeding similarly to Reference [1] we obtain

$$
\begin{aligned}
G\left(x_{i}, y_{i}, x_{j}, y_{j}\right)= & \frac{1}{4 \pi \lambda^{2}}\left\{\ln \left[\frac{\cosh \left[\pi\left|x_{i}-x_{j}\right| / b\right]-\cos \left[\pi\left(y_{i}+y_{j}\right) / b\right]}{\cosh \left[\pi\left|x_{i}-x_{j}\right| / b\right]-\cos \left[\pi\left(y_{i}-y_{j}\right) / b\right]}\right]\right. \\
& -\ln \left[\frac{\cosh \left[\pi\left(a-x_{i}-x_{j}\right) / b\right]-\cos \left[\pi\left(y_{i}+y_{j}\right) / b\right]}{\cosh \left[\pi\left(a-x_{i}-x_{j}\right) / b\right]-\cos \left[\pi\left(y_{i}-y_{j}\right) / b\right]}\right] \\
& \left.-\ln \left[\frac{\cosh \left[\pi\left(a+x_{i}+x_{j}\right) / b\right]-\cos \left[\pi\left(y_{i}+y_{j}\right) / b\right]}{\cosh \left[\pi\left(a+x_{i}+x_{j}\right) / b\right]-\cos \left[\pi\left(y_{i}-y_{j}\right) / b\right]}\right]\right\} .
\end{aligned}
$$

In the short range distance limit, London theory must be regularized, since $G$ is infinite for $(x, y)=\left(x^{\prime}, y^{\prime}\right)$. We overcome this difficulty by introducing a sharp cutoff in which $\left|x-x^{\prime}\right|$ is replaced by $\xi$. By taking the limit $(b / \pi \xi) \gg 1$ we obtain

$$
\begin{aligned}
G\left(x_{i}, y_{i}\right)= & \frac{1}{4 \pi \lambda^{2}}\left\{\ln \left[\frac{(\pi \xi / b)^{2}+4 \sin ^{2}\left(\pi y_{i} / b\right)}{(\pi \xi / b)^{2}}\right]\right. \\
& -\ln \left[\frac{\cosh \left[\pi\left(a-2 x_{i}\right) / b\right]-\cos \left(2 \pi y_{i} / b\right)}{\cosh \left[\pi\left(a-2 x_{i}\right) / b\right]-\cos (\pi \xi / b)}\right] \\
& \left.-\ln \left[\frac{\cosh \left[\pi\left(a+2 x_{i}\right) / b\right]-\cos \left(2 \pi y_{i} / b\right)}{\cosh \left[\pi\left(a+2 x_{i}\right) / b\right]-\cos (\pi \xi / b)}\right]\right\} .
\end{aligned}
$$

In Reference [2] it was also determined the Gibbs free energy of the vortex system. We found

$$
\begin{aligned}
\mathcal{G}= & \frac{\Phi_{0}^{2}}{8 \pi A} \sum_{i=1}^{N} \sum_{j=1}^{N} G\left(x_{i}, y_{i}, x_{j}, y_{j}\right)+\frac{\Phi_{0} H}{4 \pi A} \sum_{i=1}^{N} \frac{\cosh \left[\left(y_{i}-b / 2\right) / \lambda\right]}{\cosh (b / 2 \lambda)} \\
& +\frac{\Phi_{0} H}{\pi^{2} A} \sum_{i=1}^{N} \sum_{m=0}^{\infty} \frac{1}{\alpha_{2 m+1}^{2}} \frac{1}{2 m+1} \sin \left[\frac{(2 m+1) \pi y_{i}}{b}\right] \frac{\cosh \left(\alpha_{2 m+1} x_{i} / \lambda\right)}{\cosh \left(\alpha_{2 m+1} a / 2 \lambda\right)} \\
& +\frac{H^{2}}{8 \pi}\left\{1-\frac{\tanh (b / 2 \lambda)}{(b / 2 \lambda)}-\frac{8}{\pi^{2}} \sum_{m=0}^{\infty}\left[\frac{1}{(2 m+1) \alpha_{2 m+1}}\right]^{2} \frac{\tanh \left(\alpha_{2 m+1} a / 2 \lambda\right)}{\left(\alpha_{2 m+1} a / 2 \lambda\right)}\right\} \\
& -\frac{N \Phi_{0} H}{4 \pi A} .
\end{aligned}
$$
by

Finally, we quote the result for the magnetization $4 \pi M=H-B$, where $B$ is the average magnetic induction and is given

$$
\begin{aligned}
B= & \frac{\Phi_{0}}{A}\left\{N-\sum_{i=1}^{N} \frac{\cosh \left[\left(y_{i}-b / 2\right) / \lambda\right]}{\cosh (b / 2 \lambda)}\right. \\
& \left.-\frac{4}{\pi} \sum_{i=1}^{N} \sum_{m=0}^{\infty} \frac{1}{\alpha_{2 m+1}^{2}} \frac{1}{(2 m+1)} \sin \left[\frac{(2 m+1) \pi y_{i}}{b}\right] \frac{\cosh \left(\alpha_{2 m+1} x_{i} / \lambda\right)}{\cosh \left(\alpha_{2 m+1} a / 2 \lambda\right)}\right\} \\
& +H\left\{\frac{\tanh (b / 2 \lambda)}{(b / 2 \lambda)}+\frac{8}{\pi^{2}} \sum_{m=0}^{\infty}\left[\frac{1}{(2 m+1) \alpha_{2 m+1}^{2}}\right]^{2} \frac{\tanh \left(\alpha_{2 m+1} a / 2 \lambda\right)}{\left(\alpha_{2 m+1} a / 2 \lambda\right)}\right\} .
\end{aligned}
$$


These formulae constitute the basis of our discussion of the vortex configurations, energy, magnetization, and the depairing critical current density.

\section{Critical Fields and Vortex Configu- rations}

The calculation of the applied field sufficient for the entrance of another vortex, provided that $N$ vortices have already penetrated in the sample, was done considering that the Gibbs free energy is continuous at the transition from $N$ - to $(N+1)$-vortex state,

$$
\mathcal{G}_{N}-\mathcal{G}_{N+1}=0 \text {. }
$$

The root of this equation determines a series of critical fields $H_{s N}$ (with $H_{s 1}=H_{c 1}$, the lower critical field). Since the vortex positions $\left(x_{i}, y_{i}\right)$ depend on the applied field $H$, (8) is a transcendental equation. So we can solve it only numerically. We have done this by using the routine ZBRENT
[5]. For a fixed value of $H$, the vortex state is determined by minimizing the free energy $\mathcal{G}_{N}(H)$. This process is repeated for several values of $H$ until (8) converges to a fixed value $H_{s N}$.

The minimization of $\mathcal{G}_{N}(H)$ is carried out by using the simulated annealing method [6]. Within this method several vortex configurations are randomly generated. By using the Metropolis algorithm, the simulated annealing method lead the system to the most promising configuration. This method, carried out with sufficient care, usually leads us to the global minimum vortex configuration.

\section{Results}

In what follows, energy is in units of $\mathcal{G}_{0}=\left(\Phi_{0} / 4 \pi \lambda^{2}\right)^{2}$ and fields in units of $H_{0}=\Phi_{0} / 4 \pi \lambda^{2}$. The vortex configurations were determined for the case $a=b=0.5 \lambda$ and $\kappa=100$, for several values of $N$. The results are shown in Fig. 1. Notice that in all cases there is at least one symmetry axis, namely, the $y$-axis.
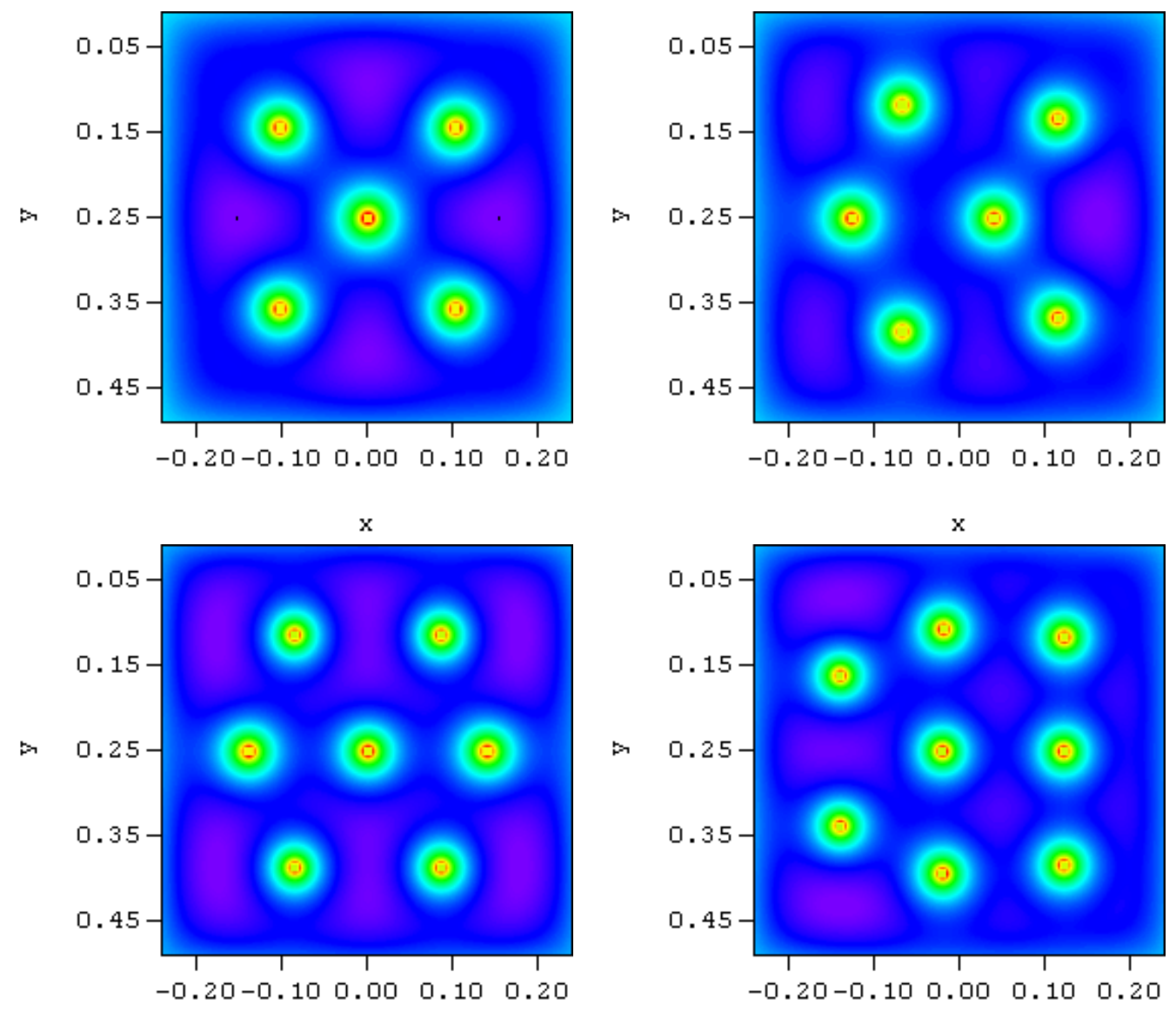

$\mathrm{x}$

$\mathrm{x}$

Figure 1. The local magnetic field profile for $N=5,6,7,8$ vortices.

Using the same value of $\kappa$ as quoted above we determined the Gibbs free energy for three different square dimension $0.5 \lambda \times 0.5 \lambda, 0.45 \lambda \times 0.45 \lambda, 0.4 \lambda \times 0.4 \lambda$. The results are illustrated in Fig. 2. As can be seen, by decreasing the square size, the energy tends to increase more slowly as a function of the $H$ field. Nevertheless, in all cases stud- 
ied the free energy is far from achieving a constant value as $H$ increases. When $\mathcal{G}$ goes to a constant the superconductor returns to its normal state since the magnetization $M \propto \partial \mathcal{G} / \partial H=0$. This behavior of the energy is more evident for the cases in which the size of the square is of order $\xi$ [7]. This scenario could never be achieved in the present context we study the vortex lattice, since the density of vortices would be so high that London theory breaks down.

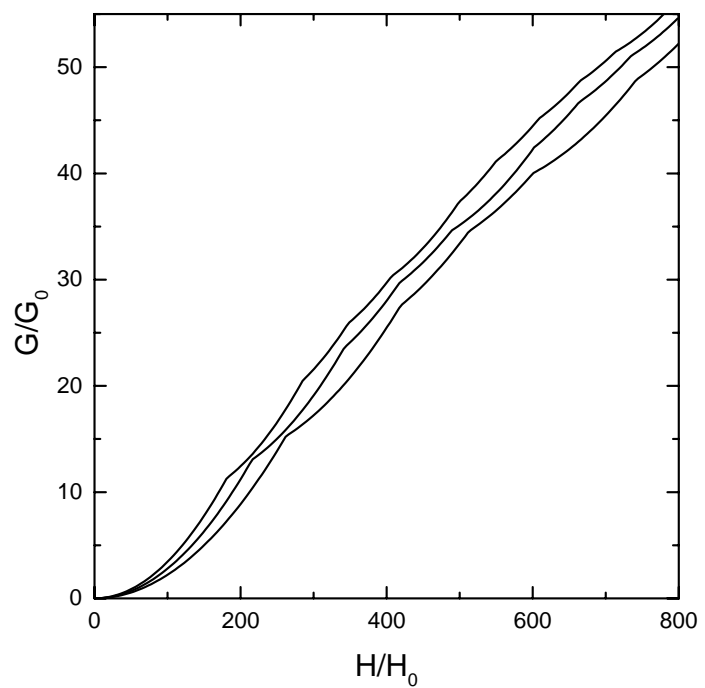

Figure 2. The Gibbs free energy as a function of the applied field $H$; we have subtracted from the free energy its value in the Meissner state, that is, the last but one term in equation (6). The discontinuities of the energy signals the entrance of another vortex.

The magnetization was also calculated for a $0.5 \lambda \times 0.5 \lambda$ square. The result is shown in Fig. 3. Notice that the magnetization shows a series of sharp peaks. Each one signals the entrance of a new vortex. In contrast to what occurs with a rectangular superconductor [2], the peaks are much more intense and the distances between the critical fields are much larger. Thus, the energy cost in creating a new vortex is much higher. Notice also that the sequence of peaks are less regular than for a rectangular superconductor [2].

Finally, we calculated the depairing critical current density. Although the geometry does not allow us to consider the vortex as a circle, we took this approximation. So it is sufficient to evaluate one component of the current density such as $J_{y}\left(x_{j}+\xi, y_{j}\right)$; at $\left(x_{j}+\xi, y_{j}\right), J_{x}$ vanishes since the vortex is circular. In the calculation, we also included the Meissner currents. Because of the proximity with the surface, the value of the current density near the core may change its value from vortex to vortex. This makes difficult the comparison with the depairing critical current density of an bulk superconductor, $J_{0}=c \Phi_{0} / 8 \pi^{2} \lambda^{2} \xi$ [8]. To overcome this difficulty, we made a global comparison by calculating $\Delta J=\sum_{j=1}^{N} J_{y}\left(x_{j}+\xi, y_{j}\right)-N J_{0}$. The term $N J_{0}$ represents the sum of all current densities as if no surface is present. The other term is its equivalent one for a rectangular superconductor. In Fig. 4 we present the result for $\Delta J$.
Notice that as the applied field increases, the difference in the current density decreases. In addition, the current density shows a series of discontinuities (matching effect), similar to what occurs with the magnetization. We must address that this behavior has been observed in the transport critical current density [1], but not the more fundamental quantity, the depairing critical current density.

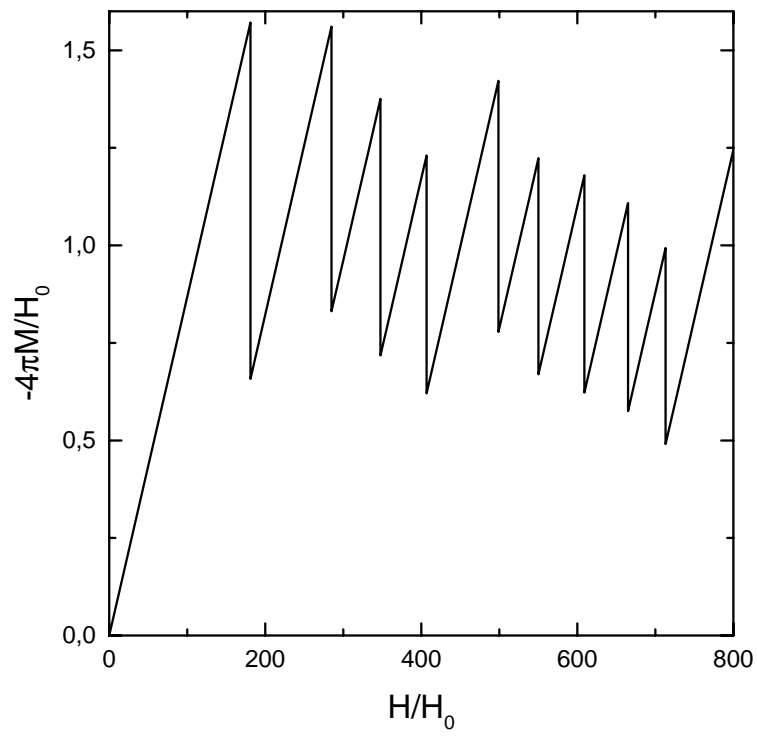

Figure 3. Magnetization as function of the applied field $H$.

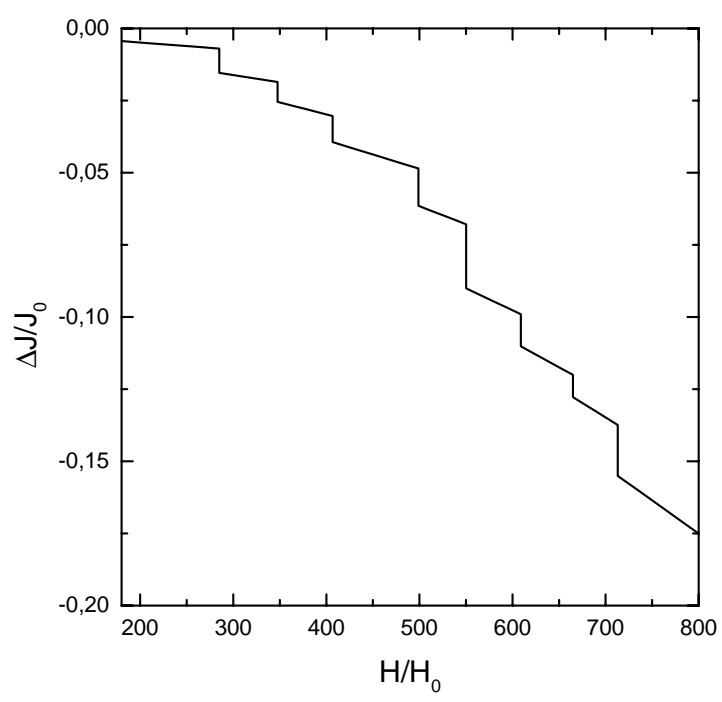

Figure 4. Depairing critical current density as a function of the applied field $H$.

TMO thanks the Brazilian Agency FAPESP for financial support. 


\section{References}

[1] Y. Mawatari and K. Yamafuji, Physica C 228, 336 (1994).

[2] E. Sardella, M. M. Doria, and P. R. S. Netto, Phys. Rev. B 60, 13158 (1999).

[3] A. S. Mel'nikov, I. M. Nefdov, D. A. Ryzhov, I. A. Shereshevskii, V. M. Vinokur, and P. P. Vysheslavtsev, Phys. Rev. B 65, 140503 (2002).

[4] M. M. Doria and G. F. Zebende, B. J. Phys. 32, 685 (2002).

[5] W. H. Press, Saul A. Teukolsky, W. T. Vetterling, and B. P.
Flannery, Numerical Recipes in FORTRAN, Cambridge University Press, Cambridge, (1992).

[6] Goffe, William L. (1996) SIMANN: A Global Optimization Algorithm using Simulated Annealing, Studies in Nonlinear Dynamics \& Econometrics: Vol. 1: No. 3, Algorithm 1.

[7] B. J. Baelus, S. V. Yampolskii, F. M. Peeters, E. Montevecchi, and J. O. Indekeu Phys. Rev. B 65, 24510 (2002).

[8] T. P. Orlando and K. A. Delin, Foundations of Applied Superconductivity, Addison-Wesley Publishing Company, page 266 (1990). 